\title{
Artery of Percheron occlusion: role of diffusion-weighted imaging in the early diagnosis
}

\author{
Murali Krishna Menon ${ }^{1}$, Suma Mariam Jacob², Muhammed Jasim Abdul Jalal ${ }^{3}$ \\ ${ }^{1}$ Department of Neurology, Lakeshore Hospital and Research Centre, Ernakulam 682040, Kerala, India. \\ ${ }^{2}$ Department of Radiology, Lakeshore Hospital and Research Centre, Ernakulam 682040, Kerala, India. \\ ${ }^{3}$ Department of Family Medicine, Lakeshore Hospital and Research Centre, Ernakulam 682040, Kerala, India.
}
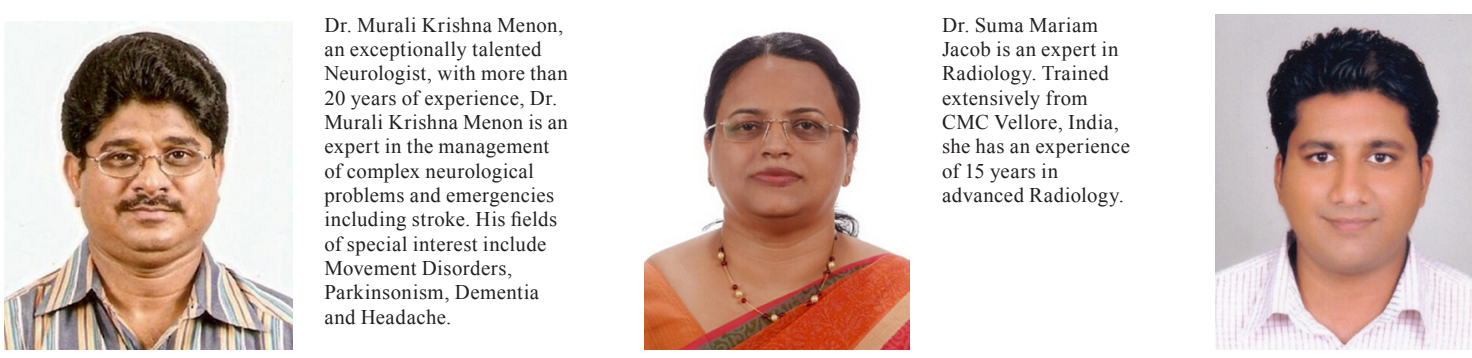

Dr. Muhammed Jasim Abdul Jalal, a Family Physician, trained in General Medicine, General Surgery, Women and Child health and Preventive Medicine. He of medical care with preventive and holistic preventive and holistic approach. He has special nterest in Clinica

\section{A B S T R A C T}

Bilateral thalamic infarcts have a low frequency among different subtypes of strokes. Since it does not involve a particular vascular territory, it therefore usually involves the occlusion of the artery of Percheron (AOP). Here we report a 79-year-old right-handed Parkinsonian female patient, who was found unresponsive in bed. On examination, the patient was drowsy with a Glasgow Coma Score (GCS) of 10/15 (E2M5V3). She had absent doll's eye response with anisocoric pupils and intermittent vertical gaze palsy. Although the patient had no apparent motor deficits, she was in a state of persistent somnolence with memory impairment and lack of initiative. Diffusion-weighted magnetic resonance imaging (MRI) of the brain showed focal areas of restricted diffusion in the medial part of the thalami bilaterally and the rostral part of mid-brain (right $>$ left) (bilateral paramedian thalamic with mid-brain pattern), suggestive of a hyper-acute infarct in the territory of AOP. The patient was anticoagulated with $40 \mathrm{mg}$ subcutaneous low molecular weight heparin and was started on double anti-platelets along with supportive measures. The level of consciousness is improved at a slow rate to a GCS of $12 / 15$ (E4M5V3). The patient had marked abulia with periods of drowsiness interspersed with periods of restlessness and uttering of abnormal sounds, but she was able to execute simple commands. In conclusion, occlusion of the AOP is a rare cause of coma in elderly patients. Diffusion-weighted MRI is the imaging modality of choice for early diagnosis. Early diagnosis of AOP occlusion may lead to favorable outcomes.

Key words: Artery of Percheron; bilateral thalamic infarcts; coma; diffusion-weighted imaging; thalamic dementia

\section{INTRODUCTION}

Bilateral thalamic infarcts have a low frequency among different subtypes of strokes. These infarcts involve the medial aspects of the thalamus in a relatively symmetrical pattern with or without simultaneous involvement of the rostral mid-brain bilaterally. Since it does not involve any of the particular vascular territories, this usually points

Corresponding Author: Dr. Muhammed Jasim Abdul Jalal,

Department of Family Medicine, Lakeshore Hospital and Research Centre, Maradu, NH 47-Byepass, Ernakulam 682040, Kerala, India.E-mail: poolspuff@gmail.com

\begin{tabular}{|l|l|}
\hline \multicolumn{2}{|c|}{ Access this article online } \\
\hline Quick Response Code: & Website: \\
\hline & www.nnjournal.net \\
\hline
\end{tabular}

out to the occlusion of the artery of Percheron (AOP). ${ }^{[1]}$ AOP is a solitary arterial trunk which arises from one of the proximal segments of the posterior cerebral artery and supplies the thalamus and certain structures of the rostral midbrain. The low sensitivity of computed tomography (CT) makes AOP infarction diagnosis difficult. Diffusion-weighted magnetic resonance imaging (MRI) is the imaging modality of choice ${ }^{[1]}$ However, AOP is rarely visualized on magnetic resonance angiography, and lack of visualization does not exclude its presence. ${ }^{[1]}$ The incidence of AOP occlusion is rare, and early diagnosis

This is an open access article distributed under the terms of the Creative Commons Attribution-NonCommercial-ShareAlike 3.0 License, which allows others to remix, tweak, and build upon the work non-commercially, as long as the author is credited and the new creations are licensed under the identical terms.

For reprints contact: service@oaepublish.com

Cite this article as: Menon MK, Jacob SM, Jalal MJ. Artery of Percheron occlusion: role of diffusion-weighted imaging in the early diagnosis. Neuroimmunol Neuroinflammation 2016;3:14-6.

Received: 09-07-2015; Accepted: 01-10-2015 
is challenging. It varies $0.1-2 \%$ in all ischemic strokes and $4-18 \%$ in thalamic infarction. ${ }^{[2]}$ Here, we report a case of bilateral thalamic infarct due to occlusion of the AOP.

\section{CASE REPORT}

A 79-year-old right-handed Parkinsonian female was found unresponsive in her bed at home. She was last seen normal approximately $8 \mathrm{~h}$ prior to her admission. There was no recent history of fever, headache, seizure, trauma, and known exposure to toxic substances. There was no history of any memory impairment or dementia. On examination, the patient was drowsy with a Glasgow Coma Score (GCS) of 10/15 (E2M5V3). She had absent doll's eye response with anisocoric pupils and intermittent vertical gaze palsy. The deep tendon reflexes were present and symmetric. Babinski sign was present bilaterally. Although the patient had no apparent motor deficits, she was in a state of persistent somnolence with memory impairment and lack of initiative.

\section{Investigations}

Laboratory findings including blood glucose, complete blood count, serum electrolytes, liver and renal function tests, thyroid function tests, arterial blood gas, and ammonia were unremarkable. Electrocardiogram showed normal sinus rhythm.

\section{Imaging}

The initial CT showed no obvious brain lesion. MRI of the brain showed focal areas of restricted diffusion [Figures 1 and 2] in the medial part of the thalami bilaterally (bilateral paramedian thalamic with mid-brain pattern), and in the rostral part of mid-brain (right $>$ left). Echo-planar two-dimensional perfusion imaging revealed areas of decreased perfusion in the areas of restricted diffusion [Figure 3]. There were V-shaped hyper-intense signal areas in the pial surface of the midbrain adjacent to the interpeduncular fossa, and therefore, no abnormal signs in this region on the T2-weighted scan [Figure 4]. These imaging findings were suggestive of a hyper-acute infarct. MRI data demonstrated patent basilar tip and posterior cerebral arteries [Figure 5]. Hence, the possibility of hyper-acute infarct in the territory of AOP was considered.

\section{Treatment}

The patient was anticoagulated with $40 \mathrm{mg}$ subcutaneous low molecular weight heparin. The level of consciousness is improved to a GCS of 12/15 (E4M5V3). The patient had marked abulia with periods of drowsiness interspersed with periods of restlessness and uttering of abnormal sounds, but she was able to execute simple commands. The patient is currently under our follow-up. She is on anticoagulation. Her consciousness is gradually improved. However, her memory impairment was still persisting as her mini

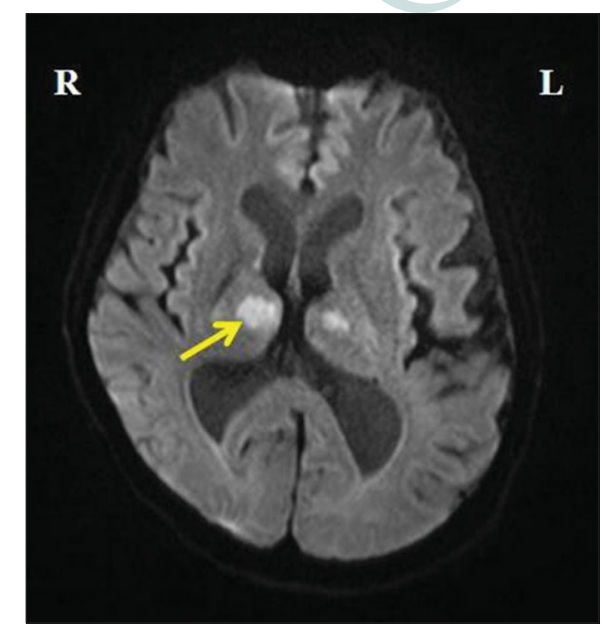

Figure 1: Magnetic resonance imaging of the brain (T1-weighted sequence) showing focal areas of restricted diffusion in the medial part of the thalami bilaterally and in the rostral part of mid-brain (right > left)

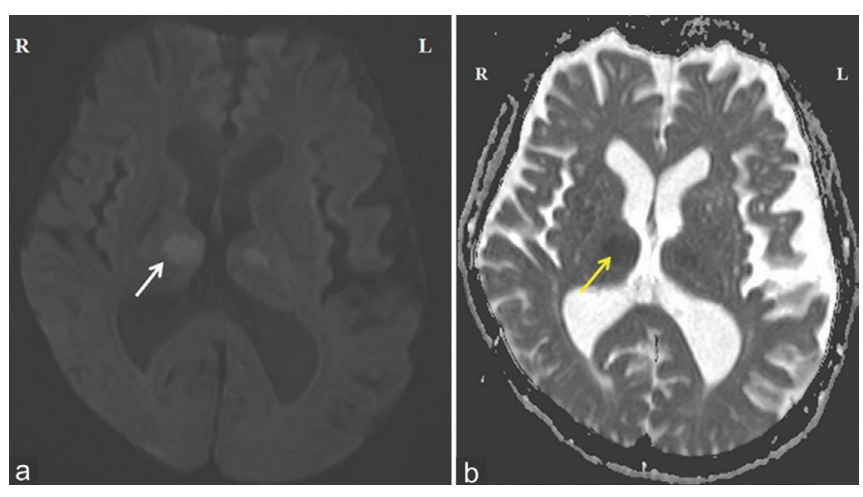

Figure 2: Magnetic resonance imaging of the brain showing focal areas of restricted diffusion in the medial part of the thalami bilaterally and in the rostral part of mid-brain (right > left). (a) Diffusion-weighted imaging sequence; (b) apparent diffusion co-efficient sequence

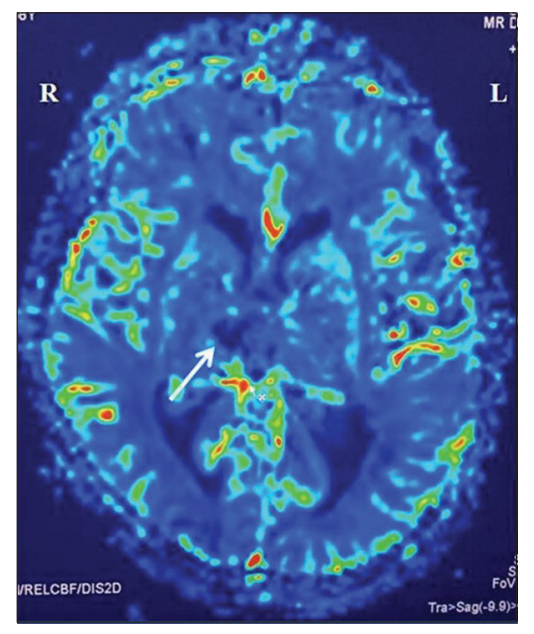

Figure 3: Echo-planar two-dimensional perfusion imaging revealing areas of decreased perfusion in the areas of restricted diffusion

mental state examination score was 23 of 30 .

\section{DISCUSSION}

Our case illustrates the importance of consideringischemic stroke in the AOP territory as one of the differential diagnosis of acute disturbance of consciousness in the elderly. Bithalamic paramedian infarcts due to occlusion of AOP presents with vertical gaze palsy (65\%), memory 


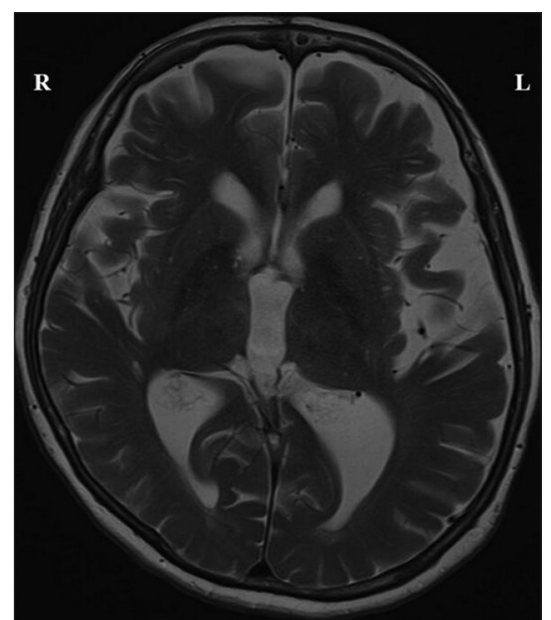

Figure 4: Magnetic resonance imaging brain T2-weighted sequences showing $V$-shaped hyper-intense signal areas in the pial surface of the midbrain adjacent to the interpeduncular fossa without any abnormal signs in this region

impairment (58\%), confusion (53\%), and coma (42\%). ${ }^{[2]}$ Vertical gaze palsy is due to the disruption of the cortical input that traverses the thalamus to reach the rostral interstitial medial longitudinal fasciculus. ${ }^{[3]}$ Memory impairment, confusion, and coma classically seen in bilateral thalamic infarction, often called together as "thalamic dementia" is explained by the involvement of the reticular activating system and the disrupted connections between the thalamus and the cortex. ${ }^{[4]}$ Thalamic dementia does not develop in case of unilateral lesions.

These patients must be differentiated from those with "top of the basilar artery" syndrome and deep cerebral venous thrombosis (DCVT). ${ }^{[5,6]}$ "Top of the basilar artery" syndrome tends to involve the superior cerebellar artery and posterior cerebral artery territories. MRI showing patent basilar tip and posterior cerebral arteries exclude this diagnosis in our patient. MRI pattern does not confine to a typical arterial territory in DCVT.

Percheron described four normal variations of the neurovascular anatomy of the thalamus and the midbrain. ${ }^{[2]}$ The medial part of the thalamus is supplied from the posterior circulation via the perforating thalamic arteries, which are also known as the paramedian arteries. ${ }^{[2]}$ Occlusion of AOP causes a bilateral paramedian thalamic and rostral midbrain infarction. Most of the AOP infarction is due to small vessel occlusion or cardiac embolism. ${ }^{[5]}$

Successful tissue plasminogen activator therapy for AOP occlusion is reported in literature, ${ }^{[7]}$ but our patient was outside the treatment time window on the initial
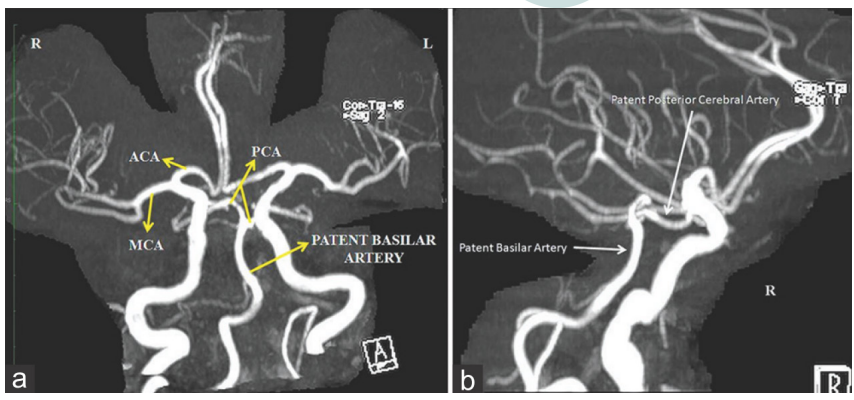

Figure 5: Magnetic resonance angiography showing patent basilar tip and posterior cerebral arteries. (a) Anterior view; (b) lateral view right side

presentation. Long-term anticoagulant therapy is the current treatment strategy suggested for AOP occlusion. ${ }^{[7]}$

In comparison to ischemic lesions of other cortical-subcortical structures, thalamic stroke has a lower mortality rate and a better prognosis as far as the recovery of motor deficits is concerned. On the contrary, the neuropsychological deficits in terms of memory, cognition, emotional response and behavior tend to persist, and interfere with the social and professional life of the patient.

In conclusion, occlusion of the AOP is a rare cause of coma in elderly patients. Diffusion-weighted MRI is the imaging modality of choice for early diagnosis. Early diagnosis of AOP occlusion may lead to favorable outcomes.

\section{Financial support and sponsorship}

Nil.

\section{Conflicts of interest}

There are no conflicts of interest.

\section{REFERENCES}

1. Chang YM, Fan YK. Artery of percheron occlusion in an elderly male: a case report. J Clin Med Res 2015; 7:126-8.

2. Lamot U, Ribaric I, Popovic KS. Artery of Percheron infarction: review of literature with a case report. Radiol Oncol 2015;49:141-6.

3. Monet P, Monet P, Garcia PY, Saliou G, Spagnolo S, Desblache J, Franc J, Vallée JN, Deramond H, Lehmann P. Bithalamic infarct: is there an evocative aspect? Radioclinical study. Rev Neurol (Paris) 2009;165:178-84.

4. Lazzaro NA, Wright B, Castillo M, Fischbein NJ, Glastonbury CM, Hildenbrand PG, Wiggins RH, Quigley EP, Osborn AG. Artery of percheron infarction: imaging patterns and clinical spectrum. AJNR Am J Neuroradiol 2010;31:1283-9.

5. Rodriguez EG, Lee JA. Bilateral thalamic infarcts due to occlusion of the Artery of Percheron and discussion of the differential diagnosis of bilateral thalamic lesions. J Radiol Case Rep 2013;7:7-14.

6. Kostanian V, Cramer SC. Artery of Percheron thrombolysis. AJNR Am J Neuroradiol 2007;28:870-1.

7. Li X, Agarwal N, Hansberry DR, Prestigiacomo CJ, Gandhi CD. Contemporary therapeutic strategies for occlusion of the artery of Percheron: a review of the literature. J Neurointerv Surg 2015;7:95-8. 